\title{
Assessing risk factors for major adverse cardiovascular and cerebrovascular events during the perioperative period of carotid angioplasty with stenting patients
}

\author{
JUAN LIU, ZHI-QIANG XU, MIN CUI, LING LI, YONG CHENG and HUA-DONG ZHOU \\ Department of Neurology and Center for Clinical Neuroscience, Daping Hospital, \\ Third Military Medical University, Chongqing 400042, P.R. China
}

Received December 8, 2015; Accepted May 18, 2016

DOI: $10.3892 / \mathrm{etm} .2016 .3360$

\begin{abstract}
Carotid atherosclerotic stenosis is a risk factor for ischemic stroke. The rapid development of neuroimaging techniques had led to carotid angioplasty with stenting (CAS) becoming a useful, effective and minimally invasive method for the treatment of extracranial carotid artery stenosis. The aim of the present study was to identify independent risk factors to predict perioperative major adverse cerebral and cardiovascular events for CAS patients and establish a risk evaluation model. Consecutive patients treated with a standardized CAS procedure were enrolled in the present study. The patients included underwent independent neurological evaluation prior to and after the procedure and at 30 days. The rates of transient ischemic attack, stroke, myocardial infarction and mortality were recorded. A relative regression model was established to evaluate risk factors of perioperative major adverse cardiac and cerebrovascular events (MACCE). In total, 403 subjects treated with CAS were enrolled into the study at a baseline MACCE rate of $8.19 \%$, whereas the overall stroke, myocardial infarction and mortality rate at 30 days was $3.97 \%$. The multiple regression analysis revealed that certain factors significantly predicted the 30-day risk of treatment-related MACCE. These factors included age of $\geq 70$ years, ulcerative plaque, severe carotid stenosis, bilateral carotid artery stenting and hemodynamic depression following CAS. The MACCE risk prediction model and risk score system were subsequently established. In conclusion, factors that significantly predicted the 30-day risk of MACCE of CAS included, age of $\geq 70$ years, ulcerative plaque, severe carotid stenosis, bilateral carotid artery stenting and hemodynamic depression, with hemodynamic depression being a controllable factor. The established risk score system is therefore a potentially useful tool that can be employed in the prediction of MACCE after CAS.
\end{abstract}

Correspondence to: Professor Hua-Dong Zhou, Department of Neurology and Center for Clinical Neuroscience, Daping Hospital, Third Military Medical University, 10 Changjiang Branch Road, Chongqing 400042, P.R. China

E-mail: zhouhuad@163.com

Key words: carotid angioplasty, ischemic stroke, artery stenosis

\section{Introduction}

Carotid atherosclerotic stenosis is one of the main risk factor for ischemic stroke and it contributes to $>20 \%$ of incidence of ischemic stroke (1). Ischemic stroke can be prevented by treating the carotid atherosclerotic stenosis. The carotid artery intima stripping off technique remains the gold standard for the treatment of carotid atherosclerotic stenosis (1). However, in recent years, the rapid development of neuroimaging techniques has led to carotid angioplasty with stenting (CAS) becoming a simple, effective and minimally invasive method used for the treatment of extracranial carotid artery stenosis and has considerable promise to become an alternative treatment to carotid endarterectomy (CEA).

A previous study evaluated the risk of perioperative stroke, myocardial infarction, and mortaltiy associated with CAS and thus a quantitative scoring system was established (2). However, that study focused on the assessment of high-risk patients with CEA and did not include factors that cannot be controlled, such as age, gender, history of cardiovascular disease, and characteristics of lesions. Another study showed that, CAS-associated complications such as vasospasm, low blood flow dynamics change, acute in-stent thrombosis, and plaque prolapse, increase the episodes of major adverse cardiac and cerebrovascular events (MACCE) (3).

In this context, the aim of the present study was to identify the independent risk factors for MACCE, and establish a model for risk scoring for CAS. Patients underwent CAS and were followed up perioperatively. By considering the baseline, disease characteristics, age, gender and postoperative complications.

\section{Materials and methods}

Patients. A total of 403 subjects were included in the study. There were 298 males and 105 females, and the patient age range was 45-83 years, with an average of $66.73 \pm 7.03$ years. The patients underwent CAS in the Department of Neurology, Daping Hospital affiliated to the Third Military Medical University (Chongqing, China) between January 2010 and June 2013 were included. The present study was approved by the Medical Ethics Committee of Daping Hospital affiliated to the Third Military Medical University. The purpose 
and methods of this study were explained to the patients or their family members and written informed consent was provided to undergo relevant examinations required for the current study. The carotid atherosclerotic stenosis was measured as described in the North American Symptomatic Carotid Endarterectomy Trial (4). Diagnosis was made using head and neck computed tomography (CT) angiography and cerebrovascular digital subtraction angiography (DSA). The diagnosed carotid atherosclerotic stenosis was stratified into symptomatic carotid atherosclerotic stenosis ( $\geq 50 \%$ stenosis) and asymptomatic atherosclerotic stenosis ( $\geq 70 \%$ stenosis).

Inclusion and exclusion criteria. Inclusion criteria for the study were as described in the guidelines for carotid stenting (5): i) asymptomatic carotid atherosclerotic stenosis patients; ii) symptomatic carotid atherosclerotic stenosis patients; and iii) patients or their family members who expressed willingness to participate in the study and signed the informed consent.

Exclusion criteria for the present study were as described by Brott et al (1): i) patients with loss of ipsilateral brain function, and paralysis, who did not benefit sufficiently from CAS; ii) lesion being completely occluded and the length of the lesion being $>10 \mathrm{~mm}$, and no blood flow observed at the distal DSA; iii) ipsilateral intracranial arteriovenous malformations or aneurysms with bleeding tendency; iv) the incidence of intracerebral hemorrhage in the previous 3 months, or large cerebral infarction (CI) in the previous 4 weeks or acute myocardial infarction in the previous 2 weeks; v) severe heart, liver and kidney dysfunction; vi) contrast agent allergy and other angiographic contraindication; vii) allergy to aspirin enteric-coated tablets, clopidogrel and other anti-platelet agents; viii) combination of serial lesion and anterior circulation, posterior circulation and multiple cerebral vascular stent implantations; and ix) serious sick sinus syndrome or atrioventricular block.

Collection of relevant patient data. Information concerning gender, age, smoking status and whether the patients had diseases or disorders including hypertension, diabetes, hypercholesterolemia, coronary cardiopathy, transient ischemic attack (TIA), symptomatic carotid atherosclerosis stenosis and CI was collected from the medical record of the patients.

Basis of diagnosis of disease or disorders. One week after patients were enrolled, blood samples were collected aseptically from the elbow vein in the morning and subjected to analyses of total cholesterol, low-density lipoprotein cholesterin and blood glucose.

Diagnosis of hypertension. Diagnosis of hypertension was carried out in accordance with Lloyd-Jones et al (6). Hypertension was defined as systolic blood pressure (SBP) greater $\geq 140 \mathrm{~mm} \mathrm{Hg}$ or diastolic blood pressure (DBP) greater $\geq 90 \mathrm{~mm} \mathrm{Hg}$, or receiving medication specifically for the indication of hypertension.

Diabetes mellitus. The diagnostic criterion, i.e., diabetes mellitus, established by the American Diabetes Association (7) was adhered to in diagnosing diabetes among patients.
Diagnosis of hypercholesterolemia. The National Cholesterol Education Program Adult Treatment Panel III (8) was adhered to in order to identify hypercholesterolemia among patients. Hypercholesterolemia was diagnosed as total serum cholesterolemia $\geq 5.20 \mathrm{mmol} / 1$ (or $\geq 200 \mathrm{mg} / \mathrm{dl}$ ) or low-density lipoprotein cholesterol $\geq 3.40 \mathrm{mmol} / \mathrm{l}$ (or $\geq 130 \mathrm{mg} / \mathrm{dl}$ ).

Coronary cardiopathy. Coronary cardiopathy diagnosis was established by observing the clinical symptoms of patients and subjecting the patients to electrocardiogram (ECG), treadmill exercise, echocardiography, coronary CT angiography and coronary angiography.

Acute myocardial infarction. The diagnosis of acute myocardial infarction was as described previously (9). The patients with an increased amount of troponin than normal value with any of the symptoms of myocardial ischemia were considered to have acute myocardial infarction. Myocardial ischemia symptoms are changes in ischemic ECG, pathological Q wave of ECG, abnormalities in ventricular wall movement or myocardial inactivation (9).

Symptomatic and asymptomatic carotid atherosclerotic stenosis. Carotid atherosclerotic stenosis was considered symptomatic when the patients experienced TIA or CI in the previous 6 months (4). The carotid atherosclerotic stenosis was considered asymptomatic if it persisted without the incidence of TIA or stroke.

Diagnosis of aortic arch calcification. Aortic arch calcification was diagnosed through chest radiography (Philips Digital Radiography, Utrecht, The Netherlands). If the aortic arch was visible and $>1 \mathrm{~cm}$ in size with funicular and curved calcification, then it was considered as aortic arch calcification (10), as confirmed by two expert radiologists.

Carotid atherosclerotic plaque typing. Carotid atherosclerotic plaque typing was performed using head and neck CT angiography (Lightspeed 64 row spiral CT; GE Healthcare, Piscataway, NJ, USA, and Brilliance iCT, 256 row; Philips Healthcare, Cleveland, OH, USA). After a cervical plain scan, the scanned images were transferred to an AW4.2 processing workstation (64-slice CT) or EBW processing workstation (256iCT) and analysis of multiplanar reformation, maximum intensity projection, volume rendering, vessels was conducted and the carotid atherosclerotic plaque $\mathrm{CT}$ values were measured. If the $\mathrm{CT}$ was $\geq 120$ Hounsfield units $(\mathrm{Hu})$, then it was considered as calcified plaque (11), whereas $\leq 120 \mathrm{Hu}$ was considered as non-calcified plaque. If the contrast agent diffused $>1 \mathrm{~mm}$ depth along the surface of the plaque in the arterial lumen, the plaques were considered ulcerative (12).

Determination of aortic arch type. Cerebral angiography was used to identify the aortic arch type, carotid artery stenosis rate, the extent of lesion, carotid artery tortuosity and carotid artery occlusion. The total arterial diameter of the left common carotid artery was taken as a reference and the vertical distance from the top of the aortic arch to the opening of the innominate artery was measured. If the measured distance was similar to the common carotid artery, it was considered a type I aortic 
arch; if the distance was 1-2 times more than that of the common carotid artery, it was considered a type II aortic arch and if the distance was $>2$ times that of the common carotid artery, it was considered a type III aortic arch (13).

Determination of carotid stenosis rate and length of lesion. The carotid artery stenosis rate was calculated as described in NASCET (4). The carotid artery stenosis rate (\%) was calculated as: (normal carotid artery diameter of stenosis - diameter of the most narrow section/diameter of the normal carotid artery in the stenosis) $x$ 100. The degree of stenosis was divided into mild (stenosis rate $0-29 \%$ ), moderate (stenosis rate $20-69 \%$ ), severe (stenosis rate $70 \%$ and above) and occlusion (stenosis rate $100 \%$ ) in which no blood flow was evident at the distal end of the occlusion. The length of the lesion was obtained by measuring the vertical scope of the plaque. If the plaque was continuous, the distance from the top of the plaque to the bottom thereof was taken to estimate the length of the lesion. If the plaque was discontinuous, the distance from the top to the bottom of each plaque was taken and a summation was made to estimate the total length of the lesion.

Carotid artery tortuosity. Carotid artery tortuosity includes 'C'-type tortuosity, 'S'-type kinking and 'O'-type coiling. 'C'-type tortuosity is characterized by vascular wavy lines (14). ' $\mathrm{S}$ '-type kinking is characterized by vascular elongation and changed angle and ' $O$ '-type coiling is characterized by excessive elongation of vascular tortuosity as ' $\mathrm{O}$ ' configuration $(15,16)$.

$C A S$. At least 3 days prior to surgery, clopidogrel $(75 \mathrm{mg} / \mathrm{l})$ and aspirin (100 mg-200 mg/l) were administered to the patients daily. Phenobarbital sodium $(0.1 \mathrm{~g})$ was administered to the patients intramuscularly $5 \mathrm{~h}$ prior to surgical resection. For 3 days after surgery, fasudil hydrochloride $(30 \mathrm{mg}$ ) was administered to the patients in $250 \mathrm{ml}$ of $0.9 \%$ saline.

Surgery was carried out by an experienced surgeon according to the standard procedure for carotid artery angioplasty and stenting (17). The patient was placed in a supine position, and after local anesthesia (1\% lidocaine hydrochloride) a Seldinger puncture of the femoral artery was performed into the $8 \mathrm{~F}$ vessel sheath with intraoperative heparinization and additional heparin (1,000 units) was also added every $1 \mathrm{~h}$. The $8 \mathrm{~F}$ femoral artery was replaced after cerebral angiography and CAS. The $8 \mathrm{~F}$ guiding catheter that was exchanged for $300 \mathrm{~mm} 0.018$ guiding wire was positioned in the common carotid artery. A distal protection device (DPD) was placed inside the distal internal carotid artery. The DPD comprised AngioGuard (Cordis, Miami Lakes, FL, USA) and Spider RX (ev3, Plymouth, MN, USA) and was used according to the blood vessel type. In cases of excessive vascular tortuosity, the DPD was not placed because it was unable to reach the distal internal carotid artery.

The diameter of general carotid and internal carotid arteries were measured prior to and after DPD implantation in patients who underwent balloon predilation or stenting. Subsequent to stenting, if the degree of improvement was not satisfactory $(>30 \%)$, balloon dilation was performed. Repeat radiography was performed to determine whether there was any vasospasm, vascular dissection or distal blood vessel embolization. The DPD was recycled, the lead wire withdrawn along with the catheter and the surgery completed.

In cases of bilateral carotid artery stenting, surgery was performed as described above and side bracket implantation was utilized. Intraoperative ECG, blood pressure (BP), and pulse oxygen saturation prior to and after balloon predilation or stent implantation was measured and BP was monitored every $2 \mathrm{~min}$. The pre- and intraoperative rate and BP were recorded according to the National Institute of Health Stroke Scale (https://www.ninds.nih.gov/doctors/NIH_Stroke_Scale. pdf). Pacemakers in patients with one were monitored.

\section{Diagnosis and removal of common complications during surgical resection}

Vasospasm. Vasospasm was diagnosed through angiography. The appearance of rough, jagged, wavy and irregular stenosis of the vascular wall was diagnosed as vascular spasm (18). Vasospasm was mostly localized in the vicinity of DPD and stent. At the end of surgery, if the vasospasm of the vascular wall was evident, papaverine hydrochloride $(30 \mathrm{mg} / 20 \mathrm{ml}$ of $0.9 \%$ saline) was intravenously administered. After $5 \mathrm{~min}$ angiography was carried out to determine whether or not the vasospasm disappeared.

Hemodynamic depression. The diagnostic criteria for hemodynamic depression were CAS intraoperative or postoperative symptomatic or non-symptomatic BP decrease (systolic BP $<90 \mathrm{mmHg}$ ) and bradycardia (heart beat rate $<60 / \mathrm{min}$ ) (3), regardless of whether booster or heart rate-increasing drugs or pacemaker were used. The pacing rate of preventive use of temporary cardiac pacing in patients was set at $60 / \mathrm{min}$. If intra- and postoperative ECG exhibited an explicit pacing signal, hemodynamic depression was considered to exist. In case of intraoperative hemodynamic depression, atropine sulfate $(0.25-0.5 \mathrm{mg})$ or dopamine hydrochloride $(150 \mathrm{mg}$ in $35 \mathrm{ml}$ of $0.9 \%$ saline) was administered. Atropine sulfate was administered in a discontinuous manner when no rebound of heart rate was observed.

Other miscellaneous complications. Acute stent thrombosis, carotid dissection, plaque prolapse, and distal vascular embolism were also observed. However, because of their extremely low incidence, they were considered for analyses in the present study.

Postoperative monitoring. Parameters such as ECG, BP and pulse oxygen saturation were monitored following surgery for $\geq 24 \mathrm{~h}$. Of these, BP was monitored once every 5-30 mins. The antihypertensive drugs were discontinued for hemodialysis patients. If the heart rate was persistent at $<60 / \mathrm{min}$, hydrochloric acid isopropyl epinephrine $(2 \mathrm{mg}$ in $500 \mathrm{ml}$ of $0.9 \%$ saline) was administered to increase the heart rate. If systolic BP was $<90 \mathrm{mmHg}$, dopamine hydrochloride or a combination of dopamine hydrochloride and hydroxylamine was administered. Patient consciousness and physical activity were continuously monitored, and if abnormal, the patients' state was evaluated through analyses of markers of myocardial injury, ECG, magnetic resonance imaging or CT examinations. The patients were treated with clopidogrel $(75 \mathrm{mg} /$ day $)$ and aspirin (100-200 mg/day) for $\geq 3$ months. 
Table I. Baseline characteristics and imaging features of CAS patients.

\begin{tabular}{|c|c|}
\hline Characteristics & $\begin{array}{l}\text { Numerical value } \\
\qquad(\mathrm{n}=403)\end{array}$ \\
\hline \multicolumn{2}{|l|}{ Baseline characteristics } \\
\hline \multicolumn{2}{|l|}{ Demographic data } \\
\hline Age (years old, mean \pm SD) & $66.73 \pm 7.03$ \\
\hline 80 years old, no. $(\%)$ & $5(1.24)$ \\
\hline 70-80 years old, no. (\%) & $150(37.22)$ \\
\hline $60-70$ years old, no. $(\%)$ & $162(40.20)$ \\
\hline$\sim 60$ years old, no. $(\%)$ & $86(21.34)$ \\
\hline Male, no. (\%) & $298(73.95)$ \\
\hline Female, $(\%)$ & $105(26.05)$ \\
\hline \multicolumn{2}{|l|}{ Vascular risk factors } \\
\hline Hypertension, no. (\%) & $266(66.00)$ \\
\hline Diabetes mellitus, no. (\%) & $108(26.80)$ \\
\hline Hypercholesterolemia, no. (\%) & $88(21.84)$ \\
\hline Smoking, no. (\%) & $159(39.45)$ \\
\hline Coronary cardiopathy, no. (\%) & $102(25.31)$ \\
\hline Symptomatic carotid stenosis & $265(65.76)$ \\
\hline TIA, no. (\%) & $128(31.76)$ \\
\hline Cerebral infarction, no. (\%) & $137(34.00)$ \\
\hline \multicolumn{2}{|l|}{ Imaging features } \\
\hline Aortic arch calcification, no. (\%) & $86(21.34)$ \\
\hline Calcified plaque, no. (\%) & $94(23.33)$ \\
\hline Ulcer type plaque, no. (\%) & $102(25.31)$ \\
\hline \multicolumn{2}{|l|}{ Aortic arch typing } \\
\hline Aortic arch type I, no. (\%) & $114(28.29)$ \\
\hline Aortic arch type II, no. (\%) & $240(59.55)$ \\
\hline Aortic arch type III, no. (\%) & $49(12.16)$ \\
\hline Carotid artery tortuosity, no. (\%) & $217(53.85)$ \\
\hline \multicolumn{2}{|l|}{ Carotid artery stenosis } \\
\hline$\geq 70 \%$, no. $(\%)$ & $275(68.24)$ \\
\hline$<70 \%$, no. $(\%)$ & $128(31.76)$ \\
\hline \multicolumn{2}{|l|}{ Lesion length } \\
\hline$\geq 15 \mathrm{~mm}$, no. $(\%)$ & $121(30.02)$ \\
\hline$<15$ mm, no. $(\%)$ & $282(69.98)$ \\
\hline Contralateral carotid occlusion, no. (\%) & $16(3.97)$ \\
\hline Lesions carotid bifurcation, no. (\%) & $371(92.06)$ \\
\hline
\end{tabular}

$\mathrm{SD}$, standard deviation; TIA, transient ischemic attack.

Postoperative follow-up. The in-patients were monitored in the hospital whereas discharged patients were monitored when they visited the outpatient facility and via telephone enquiries for a period of 30 days post-surgery. The MACCE were considered when the patients experienced TIA, ischemic or hemorrhagic stroke, acute myocardial infarction or mortality during the postoperative monitoring period. Manifestation of stroke observed was stratified into large and minor, large stroke was 4-5 points on the modified Rankin Scale (mRS) (19) and
Table II. Post-operative adverse cardiovascular and cerebrovascular events in CAS patients.

\begin{tabular}{lr} 
Cerebral vascular event & Values, n=403 \\
\hline TIA, no. (\%) & $17(4.22)$ \\
Stroke of any cause, no. (\%) & $15(3.72)$ \\
$\quad$ Large stroke (operative side), no. (\%) & $4(0.99)$ \\
Large stroke (non-operative side), no. (\%) & $1(0.25)$ \\
Minor stroke (operation side), no. $(\%)$ & $9(2.23)$ \\
Minor stroke (non-operation side), no. (\%) & $1(0.25)$ \\
Myocardial infarction, no. $(\%)$ & $1(0.25)$ \\
Death of any cause, no. $(\%)$ & $3(0.74)$ \\
Stroke, myocardial infarction & $16(3.97)$ \\
and death, no. (\%) & $33(8.19)$ \\
TIA, stroke, myocardial & \\
infarction and death, no. $(\%)$ & \\
\hline
\end{tabular}

CAS, carotid angioplasty with stenting; TIA, transient ischemic attack.

included patients not capable of self-care, walking alone or were bedridden, coupled with the presence of aphasia or hemianopsia. Minor stroke was 2-3 points on the mRS in which patients had neurological dysfunction, self-care and absence of hemianopsia and aphasia (20).

Statistical analysis

Chi-square test and independent sample t-test. The patients were divided into the MACCE and non-MACCE groups. The mean values of continuous variables were compared using the independent t-test. In cases of categorical variables and $\geq 5$ patients, the Pearson Chi-square test was applied. If the number of patients were $<5$, the continuity corrected Chi-square test was used, and for $<1$, the Fisher's exact test was used. $\mathrm{P}<0.05$ was considered to indicate a statistically significant difference.

Unconditional logistic regression analysis. The variables which showed a significant difference in the Chi-square test and t-test were included in the dual unconditional logistic regression analysis. The variables were evaluated for their effect and influence on MACCE. The odds ratio (OR) and 95\% confidence interval (CI) were calculated for categorical variables. $\mathrm{P}<0.05$ was considered statistically significant.

Regression model construction. The regression equation was established following examination of the regression coefficient. The likelihood ratio test or Wald's Chi-square test was used for single regression coefficient hypothesis testing and the contribution of the model was determined. The test of goodness of fit of the regression model was determined using Hosmer-Lemeshow. The regression equation was expressed as: $\log (\mathrm{p})=\beta 0+\beta 1 \times 1+\ldots \beta \mathrm{n} \times \mathrm{n}$. By calculating the normalized regression coefficients of individual variables, the effect of the risk factors associated with the occurrence of MACCE was analyzed. 
Table III. Baseline characteristics of the MACCE and non-MACCE groups.

\begin{tabular}{lcccc}
\hline Characteristics & Non-MACCE group $(\mathrm{n}=370)$ & MACCE group $(\mathrm{n}=33)$ & Statistic & P-value \\
\hline Age (years old, mean \pm SD) & $69.00 \pm 6.85$ & $66.53 \pm 7.02$ & $\mathrm{t}=1.939$ & 0.053 \\
$\geq 70$ years old, no. $(\%)$ & $126(34.05)$ & $28(84.85)$ & $\chi^{2}=33.108$ & $<0.001^{\mathrm{a}}$ \\
$<70$ years old, no. $(\%)$ & $244(65.95)$ & $5(15.15)$ & & \\
Gender & & & & \\
Male, no. $(\%)$ & $277(74.86)$ & $21(63.64)$ & $\chi^{2}=1.983$ & 0.159 \\
Female, no. $(\%)$ & $93(25.14)$ & $12(36.36)$ & & \\
Hypertension, no. $(\%)$ & $241(65.14)$ & $25(75.76)$ & $\chi^{2}=1.524$ & 0.217 \\
Diabetes mellitus, no. $(\%)$ & $93(25.14)$ & $15(45.45)$ & $\chi^{2}=6.377$ & $0.012^{\mathrm{a}}$ \\
Smoking, no. $\%)$ & $145(39.19)$ & $10(42.42)$ & $\chi^{2}=0.133$ & 0.716 \\
Hypercholesterolemia, no. $(\%)$ & $78(21.08)$ & $18(54.55)$ & $\chi^{2}=1.510$ & 0.219 \\
Coronary cardiopathy, no. $(\%)$ & $84(22.70)$ & $28(84.85)$ & $\chi^{2}=16.251$ & $<0.001^{\mathrm{a}}$ \\
Symptomatic carotid stenosis & $237(64.05)$ & & $\chi^{2}=5.818$ & $0.016^{\mathrm{a}}$
\end{tabular}

(TIA, cerebral infarction), no. (\%)

${ }^{\mathrm{a}} \mathrm{P}<0.05$. SD, standard deviation; TIA, transient ischemic attack; MACCE, major adverse cardiac and cerebrovascular events.

Establishment of the risk score table. The risk score table was established from the regression model. The score was assigned as a decimal number of the OR value of the risk factor associated with the occurrence of MACCE or 0.5 after the integer. This table was used to calculate the risk score and to draw a forecast probability chart of the risk score. The score table was also used to test the forecast effect by drawing the receiver operating characteristic curve (ROC) and to calculate the area under the curve (AUC). The forecast probability chart was created through MS Excel. ROC, AUC and any other statistical tests were carried out using SPSS 18.0 (SPSS, Inc., Chicago, IL, USA).

\section{Results}

Demographics of patients. Of the 433 patients that underwent CAS, 30 patients were excluded from the present study for various reasons, including carotid and vertebral artery stenosis in multiple sites together with stent implantation (16 patients), surgical operation failure (2 patients), loss of follow-up (12 patients). A total of 403 patients were followed up for 30 days subsequent to surgery.

The patient age range was $45-83$ years with an average of $66.73 \pm 7.03$ years. The patients aged 60-70 years were higher in number (40.20\%, Table I). There were 298 males and 105 females. Of the various risk factors, hypertension was found in $66.00 \%$ of patients. Approximately $65.76 \%$ of patients had symptomatic carotid stenosis, $31.76 \%$ had TIA and $34.00 \%$ had CI.

Clinical diagnoses. Of the 403 patients, $21.34 \%$ were found to have aortic arch calcification, while $23.33 \%$ of patients had calcified plaque and $25.31 \%$ had ulcerative plaque. The type II aortic arch was identified in the majority of patients $(59.55 \%)$. The ipsilateral carotid artery tortuosity was observed in $21.34 \%$ of patients with aortic arch calcification. The majority of patients had severe carotid stenosis (68.24\%).
The carotid bifurcation region was the most frequent site of stenosis $(92.06 \%)$ and most of the lesions were $<15 \mathrm{~mm}$ (Table I).

Perioperative major adverse cardio- and cerebrovascular events. During the follow-up period, MACCE were observed in $33(8.19 \%)$ patients. Of the 33 patients, 16 patients experienced any of the following MACCE events: stroke, myocardial infarction or mortality, and TIA was experienced by 17 patients. The TIA occurred during the surgery (7 patients) and 1-3 days post-surgery in the hospital (Table II).

Comparison of baseline and clinical parameters between the MACCE and non-MACCE groups. No significant difference in the average age of patients between the MACCE and non-MACCE groups was identified. However, in the subgroup of $\geq 70$ years, MACCE was observed in $28(84.85 \%)$ of 33 patients, which showed its significant association with the incidence of MACCE $(\mathrm{P}<0.001)$. The remaining clinical parameters significantly associated with the incidence of MACCE included diabetes mellitus, coronary cardiopathy and symptomatic carotid stenosis $(\mathrm{P}<0.05$, Table III).

In addition to these results, ulcerative plaque, type III aortic arch, severe stenosis, longer lesion and carotid artery occlusion were significantly associated with the incidence of MACCE $(\mathrm{P}<0.05$, Table IV).

Intraoperative situation between MACCE and non-MACCE groups. The DPD was used in 389 (96.53\%) patients but not in 14 patients because of severe tortuosity of the internal carotid artery. A majority (18.18\%) of patients in the MACCE group received bilateral carotid stenting in comparison with patients in the non-MACCE group (5.41\%). The incidence of intraoperative hemodynamic depression was higher in the MACCE group at $31(93.94 \%)$ of 33 compared to that of the non-MACCE group at 210 of 370 patients $(56.76 \%$, Table V). 
Table IV. Comparison of imaging features of the MACCE and non-MACCE groups.

\begin{tabular}{|c|c|c|c|c|}
\hline Characteristics & Non-MACCE group $(n=370)$ & MACCE group (n=33) & Statistic & P-value \\
\hline Aortic arch calcification, no. $(\%)$ & $78(21.08)$ & $8(24.24)$ & $\chi^{2}=0.180$ & 0.671 \\
\hline Patch properties & & & $\chi^{2}=2.013$ & 0.156 \\
\hline Calcified plaque, no. (\%) & $83(22.43)$ & $11(33.33)$ & & \\
\hline Non-calcified plaque, no. (\%) & $287(77.57)$ & $22(66.67)$ & & \\
\hline Ulcer type plaque, no. (\%) & $83(22.43)$ & $19(57.58)$ & $\chi^{2}=19.794$ & $<0.001^{\mathrm{a}}$ \\
\hline Aortic arch typing & & & $\chi^{2}=7.700$ & $0.021^{\mathrm{a}}$ \\
\hline $\mathrm{I}$, no. $(\%)$ & $106(28.65)$ & $8(24.24)$ & & \\
\hline II, no. $(\%)$ & $224(60.54)$ & $16(48.49)$ & & \\
\hline III, no. (\%) & $40(10.81)$ & $9(27.27)$ & & \\
\hline Carotid artery tortuosity & $194(52.43)$ & $21(63.64)$ & $\chi^{2}=1.528$ & 0.216 \\
\hline Carotid artery stenosis & & & $\chi^{2}=4.575$ & $0.032^{\mathrm{a}}$ \\
\hline$\geq 70 \%$, no. $(\%)$ & $247(66.76)$ & $28(84.85)$ & & \\
\hline$<70 \%$, no. $(\%)$ & $123(33.24)$ & $5(15.15)$ & & \\
\hline Lesion length & & & $\chi^{2}=5.830$ & $0.016^{\mathrm{a}}$ \\
\hline$\geq 15 \mathrm{~mm}$, no. $(\%)$ & $105(28.38)$ & $16(48.48)$ & & \\
\hline$<15$ mm, no. $(\%)$ & $265(71.62)$ & $17(51.52)$ & & \\
\hline Contralateral carotid occlusion, no. (\%) & $12(3.24)$ & $4(12.12)$ & $\chi^{2}=4.151$ & $0.042^{\mathrm{a}}$ \\
\hline Lesions cross bifurcation, no. (\%) & $343(92.70)$ & $28(84.85)$ & $\chi^{2}=2.557$ & 0.110 \\
\hline
\end{tabular}

${ }^{\mathrm{a}} \mathrm{P}<0.05$. MACCE, major adverse cardiac and cerebrovascular events.

Table V. Comparison of intraoperative situation of the MACCE and non-MACCE groups.

\begin{tabular}{|c|c|c|c|c|}
\hline Characteristics & Non-MACCE group $(n=370)$ & MACCE group $(n=33)$ & Statistic & P-value \\
\hline DPD use, no. (\%) & $359(97.03)$ & $30(90.91)$ & $\chi^{2}=1.803$ & 0.179 \\
\hline \multicolumn{5}{|l|}{ DPD type, no. (\%) } \\
\hline Angioguard & $164(44.33)$ & $9(27.27)$ & $\chi^{2}=2.757$ & 0.097 \\
\hline Spider & $195(52.70)$ & $21(63.64)$ & & \\
\hline Bracket type, no. (\%) & & & $\chi^{2}=3.350$ & 0.187 \\
\hline Precise & $212(57.30)$ & $14(42.42)$ & & \\
\hline Protégé & $143(38.65)$ & $16(48.48)$ & & \\
\hline Smart Control & $15(4.05)$ & $3(9.09)$ & & \\
\hline Balloon pre expansion, no. (\%) & $47(12.70)$ & $8(24.24)$ & $\chi^{2}=3.423$ & 0.064 \\
\hline Bilateral carotid stenting, no. $(\%)$ & $20(5.41)$ & $6(18.18)$ & $\chi^{2}=8.195$ & $0.004^{\mathrm{a}}$ \\
\hline Intravascular operation time, no. $(\%)$ & & & $\chi^{2}=0.988$ & 0.320 \\
\hline$<30 \min$ & $276(74.59)$ & $22(66.67)$ & & \\
\hline$\geq 30 \mathrm{~min}$ & $94(25.41)$ & $11(33.33)$ & & \\
\hline Vasospasm, no. (\%) & $52(14.05)$ & $6(18.18)$ & $\chi^{2}=0.419$ & 0.517 \\
\hline Hemodynamic depression, no. (\%) & $210(56.76)$ & $31(93.94)$ & $\chi^{2}=15.912$ & $<0.001^{\mathrm{a}}$ \\
\hline
\end{tabular}

${ }^{\mathrm{a}} \mathrm{P}<0.05$. MACCE, major adverse cardiac and cerebrovascular events; DPD, distal protection device.

Additive effect of risk factors on the incidence of MACCE. The parameters that were significantly associated with the incidence of MACCE were included in the dual non-conditional logistic regression analysis. After removing the confounding factors, only age $\geq 70$ years, ulcerative plaque, severe stenosis, bilateral carotid stenting and hemodynamic depression were found to be independently associated with the incidence of MACCE (Table VI).

The effect of the aforementioned risk factors on the incidence of MACCE was expressed using the regression equation: 
Table VI. Association of risk factors with MACCE.

\begin{tabular}{|c|c|c|c|c|c|}
\hline Risk factor & $\mathrm{B}$ & SE & Wals & OR $(95 \% \mathrm{CI})$ & $\mathrm{P}$-value \\
\hline Age $(\geq 70)$ years & 1.609 & 0.571 & 7.949 & $4.997(1.633-15.290)$ & 0.005 \\
\hline Ulcerative plaque & 1.064 & 0.444 & 5.740 & $2.899(1.214-6.924)$ & 0.017 \\
\hline Severe stenosis & 1.245 & 0.568 & 4.807 & $3.472(1.141-10.566)$ & 0.028 \\
\hline Bilateral carotid stenting & 1.611 & 0.628 & 6.575 & $5.007(1.462-17.151)$ & 0.010 \\
\hline HD & 1.757 & 0.792 & 4.915 & $5.792(1.226-27.369)$ & 0.027 \\
\hline
\end{tabular}

MACCE, major adverse cardiac and cerebrovascular events; OR, odds ratio; CI, confidence interval; B, regression coefficient; SE, standard error; HD, haemodynamic depression.

Table VII. MACCE in perioperative period of CAS.

\begin{tabular}{lccc}
\hline Characteristics & $\mathrm{B} j$ & $\mathrm{~S} j$ & $\begin{array}{c}\text { Standardized } \\
\text { regression coefficient }\end{array}$ \\
\hline Age ( $\geq 70)$ years & 1.609 & 0.571 & 0.502 \\
Ulcerative plaque & 1.064 & 0.444 & 0.258 \\
Severe stenosis & 1.245 & 0.568 & 0.386 \\
$\begin{array}{l}\text { Bilateral carotid } \\
\text { stenting }\end{array}$ & 1.611 & 0.628 & 0.553 \\
HD & 1.757 & 0.792 & 0.760 \\
\hline
\end{tabular}

MACCE, major adverse cardiac and cerebrovascular events; HD, haemodynamic depression.

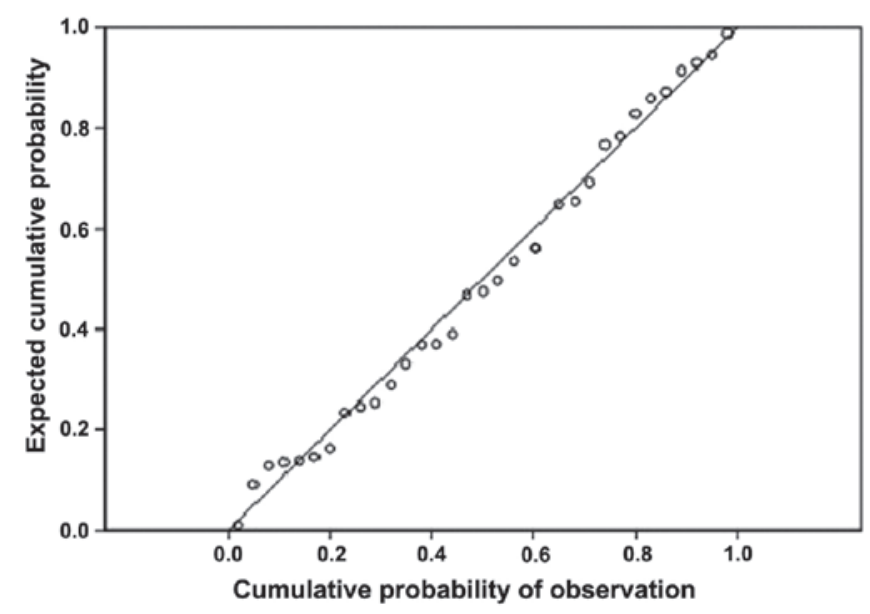

Figure 1. Standard residual cumulative probability normal P-P diagram.

Log (MACCE incidence probability) $=-8.992+1.609 \times$ age $\geq 70+1.064 \times$ ulcerative plaque $+1.245 \times$ severe stenosis $+1.611 \mathrm{x}$ bilateral carotid stenting $+1.757 \mathrm{x}$ hemodynamic depression $\left(\chi^{2}=163.478, \mathrm{P}<0.001\right)$.

These findings showed that, age, ulcerative plaque, severe stenosis, bilateral carotid stenting and hemodynamic depression were significantly associated with the incidence of MACCE. The cumulative probability plot (Fig. 1) shows that, the scatter is basically diagonal and the Hosmer-Lemeshow goodness-of-fit test indicated the model has good fitting effect.

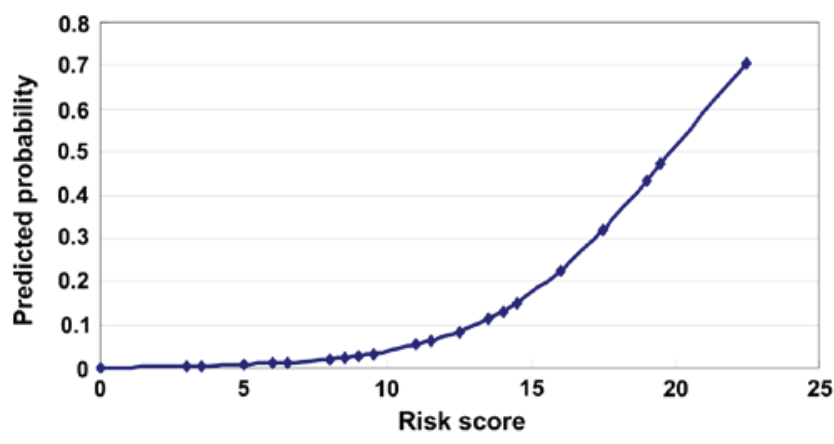

Figure 2. Forecast probability of the risk score.

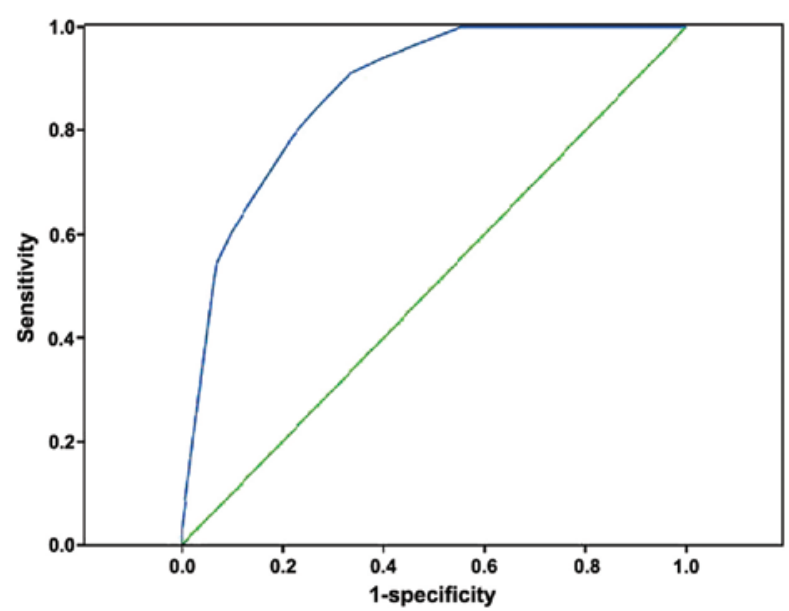

Figure 3. Forecast effect of the risk score. ROC, receiver operating characteristic.

The standard regression coefficient analysis revealed that, hemodynamic depression was the most prominent risk factor for MACCE followed by bilateral carotid artery, age $\geq 70$ years, severe stenosis and ulcerative plaque (Table VII).

Risk score table for MACCE. The risk scores of the risk factors for MACCE were: 5 points for age $\geq 70$ years, 3 points for ulcerative plaques, 3.5 points for severe stenosis, 5 points for bilateral carotid artery stenting and 6 points for hemodynamic depression.

These points were used to calculate each of the 403 patient risk scores and create the forecast probability chart. The risk 
of incidence of MACCE increased with an increasing risk score (Fig. 2).

To validate the predictive effect of risk scores, the ROC curve was drawn and AUC was found to be $0.875(\mathrm{P}<0.001$, 95\% CI: 0.825-0.925), which indicated that a good forecast effect of this risk score model for MACCE (Fig. 3).

\section{Discussion}

Currently, stroke ranks fourth among all causes of mortality in the United States and is recognized as a leading cause of serious physical and cognitive long-term disability in adults. Almost 800,000 US residents experience an incident or recurrent stroke each year (21). In China, the annual incidence of stroke is approximately 2 million, of which 1.5 million patients succumb, while the majority of survivors $(75 \%)$ become disabled. Additionally, the 5-year survival rate is $41 \%$ (22). Ischemic stroke was involved in $75-85 \%$ of all stroke incidences for which carotid atherosclerotic stenosis is one of the major contributing factors. After decades of clinical practices, CEA was considered the gold standard for the treatment of carotid atherosclerotic stenosis. Continuous advancements in the field of neuroimaging and vascular operation technology in the last decade have led to CAS potentially becoming an alternative treatment to CEA for carotid atherosclerotic stenosis (23). However, there is a lack of sufficient evidence to verify this hypothesis as the MACCE were not less in CAS than CEA during the perioperative period (24). Consequently, CAS is often recommended as an alternative treatment method to CEA only in high-risk patients (25).

Previous findings have suggested that, the incidence of ipsilateral stroke was extremely low in patients who underwent CAS in comparison with CEA (26). Therefore, we carried out risk assessment for MACCE during the perioperative period of CAS that would reduce MACCE during the perioperative period of CAS by providing intervention to complications that were significantly associated with MACCE. However, investigations pertaining to the risk of stroke, myocardial infarction and death during the CAS perioperative period are scarce (2).

In the present study, MACCE were observed in $8.19 \%$ of patients who underwent CAS, of which $3.97 \%$ experienced stroke, myocardial infarction or mortality. This incidence rate was similar to that reported worldwide (27). Although the univariate analysis revealed 11 risk factors (age, diabetes, coronary heart disease, symptomatic carotid artery stenosis, ulcerative plaque, type III aortic arch, severe carotid artery stenosis, longer lesion, carotid artery occlusion, bilateral carotid artery stenting and hypertension) associated with the incidence of MACCE, the multivariate analysis revealed the association of only five risk factors (age $\geq 70$, ulcerative plaque, bilateral carotid artery stenting, severe stenosis and hypertension) for the incidence of MACCE and a potent risk model.

In a previous study, age was found to be an independent risk factor associated with postoperative complications (28), because elderly patients are more prone to artery tortuosity and vascular calcification (29). The instability of ulcerative plaque increases the risk of TIA and ischemic stroke caused by thromboembolism during or after surgical resection (30). There is a high risk of thromboembolism during endovascular surgery where the catheter, guidewire and DPD are used to reach the lesion.
The present study also showed that bilateral carotid artery stenting is a risk factor of MACCE in CAS patients. The operation time in the blood vessels was prolonged subsequent to bilateral stent release, thus, cerebral perfusion increased, which ultimately leads to increased risk of thrombosis or cerebral hemorrhage. Severe carotid stenosis was also found to be a risk factor for MACCE. Due to preoperative cerebral hypoperfusion and ascertaining adverse collateral circulation following the discharge of bracket, severe carotid stenosis is more prone to cerebral hyperperfusion leading to hemorrhagic stroke.

Previous findings have shown that, hypertension is linked with other conditions such as coronary disease (31), type of aortic arch (32), carotid artery tortuosity, calcification (33), balloon predilation, bilateral stent implantation and other factors (34). In the present study, the interaction between these factors constitutes hypertension as a potent risk factor. A possible reason for observing CI and myocardial infarction in the current study was the decreased BP and bradycardia caused by the intra- and postoperative slowdown of clearance of emboli and plaque debris that weaken the stenosis vascular tortuosity of collateral circulation, thereby increasing the risk of cerebral ischemia and myocardial infarction.

The results of the present study established a risk score model for MACCE that may be used to quantify the risk for MACCE in CAS patients through which effective measures can be taken to reduce the incidence of MACCE in CAS patients during their perioperative period.

\section{References}

1. Brott TG, Halperin JL, Abbara S, Bacharach JM, Barr JD, Bush RL, Cates CU, Creager MA, Fowler SB, Friday G, et al; American College of Cardiology Foundation/American Heart Association Task Force on Practice Guidelines: American Stroke Association; American Association of Neuroscience Nurses; American Association of Neurological Surgeons; American College of Radiology; American Society of Neuroradiology; Congress of Neurological Surgeons; Society of Atherosclerosis Imaging and Prevention; Society for Cardiovascular Angiography and Interventions; Society of Interventional Radiology; Society of NeuroInterventional Surgery; Society for Vascular Medicine; Society for Vascular Surgery; American Academy of Neurology and Society of Cardiovascular Computed Tomography: 2011 ASA/ACCF/ AHA/AANN/AANS/ACR/ASNR/CNS/SAIP/CAI/SIR/SNIS/ SVM/SVS guideline on the management of patients with extracranial carotid and vertebral artery disease: executive summary. Stroke 42: e420-e463, 2011.

2. Setacci C, Chisci E, Setacci F, Iacoponi F, de Donato G and Rossi A: Siena carotid artery stenting score: a risk modelling study for individual patients. Stroke 41: 1259-1265, 2010.

3. Gupta R, Abou-Chebl A, Bajzer CT, Schumacher HC and Yadav JS: Rate, predictors, and consequences of hemodynamic depression after carotid artery stenting. J Am Coll Cardiol 47: 1538-1543, 2006.

4. North American Symptomatic Carotid Endarterectomy Trial Collaborators: Beneficial effect of carotid endarterectomy in symptomatic patients with high-grade carotid stenosis. N Engl J Med 325: 445-453, 1991.

5. Interventional radiology group of Radiology Branch of Chinese Medical Association: Interventional treatment for carotid stenosis (expert consensus). Zhonghua Fang She Xue Za Zhi 44: 995-998, 2010.

6. Lloyd-Jones DM, Evans JC and Levy D: Hypertension in adults across the age spectrum: Current outcomes and control in the community. JAMA 294: 466-472, 2005.

7. Expert Committee on the Diagnosis and Clasification of Diabetes Mellitus: American Diabetes Association: clinical practice recommendations 2002. Diabetes Care 25 (Suppl 1): S1-S147, 2002. 
8. National Cholesterol Education Program (NCEP) Expert Panel on Detection, Evaluation, and Treatment of High Blood Cholesterol in Adults (Adult Treatment Panel III): hird Report of the National Cholesterol Education Program (NCEP) Expert Panel on Detection, Evaluation, and Treatment of High Blood Cholesterol in Adults (Adult Treatment Panel III) final report. Circulation 106: 3143-3421, 2002.

9. No authors listed: Myocardial infarction redefined -- a consensus document of The Joint European Society of Cardiology/ American College of Cardiology Committee for the redefinition of myocardial infarction. Eur Heart J 21: 1502-1513, 2000.

10. Li J, Galvin HK, Johnson SC, Langston CS, Sclamberg J and Preston CA: Aortic calcification on plain chest radiography increases risk for coronary artery disease. Chest 121: 1468-1471, 2002.

11. Das M, Braunschweig T, Mühlenbruch G, Mahnken AH,Krings T, Langer S, Koeppel T, Jacobs M, Günther RW and Mommertz G: Carotid plaque analysis: Comparison of dual-source computed tomography (CT) findings and histopathological correlation. Eur J Vasc Endovasc Surg 38: 14-19, 2009.

12. Qi W, Guo W, Yue M and Guo Q: Use 256 slice spiral CT angiography of carotid artery to evaluate ulcer plaque. Zhongguo Jieru Yingxiang Yu Zhiliaoxue 9: 354-357, 2012.

13. Madhwal S, Rajagopal V, Bhatt DL, Bajzer CT, Whitlow P and Kapadia SR: Predictors of difficult carotid stenting as determined by aortic arch angiography. J Invasive Cardiol 20: 200-204, 2008

14. Metz H, Murray-Leslie RM, Bannister RG, Bull JW and Marshall J: Kinking of the internal carotid artery. Lancet 1: 424-426, 1961.

15. Weibel J and Fields WS: Tortuosity, coiling, and kinking of the internal carotid artery. II. Relationship of morphological variation to cerebrovascular insufficiency. Neurology 15: 462-468, 1965.

16. Jia X: DSA imaging findings, clinical features and interventional therapy of intracranial and intracranial arteriosclerosis. Third Military Medical University, Chongqing, 2010.

17. Powers CJ, Hirsch JA, Hussain MS, Patsalides AT, Blackham KA, Narayanan S, Lee SK, Fraser JF, Bulsara KR, Prestigiacomo CJ, Gandhi CD, Abruzzo T, Do HM, Meyers PM, Albuquerque FC, Frei D, Kelly ME, Pride GL and Jayaraman MV; Standards and Guidelines committee of the Society of NeuroInterventional Surgery: Standards of practice and reporting standards for carotid artery angioplasty and stenting. J Neurointerv Surg 6: 87-90, 2014.

18. Vijayvergiya R, Otaal PS, Bagga S and Modi M: Symptomatic carotid vasospasm caused by a distal-protection device during stent angioplasty of the right internal carotid artery. Tex Heart Inst J 37: 226-229, 2010.

19. van Swieten JC, Koudstaal PJ, Visser MC, Schouten HJ and van Gijn J: Interobserver agreement for the assessment of handicap in stroke patients. Stroke 19: 604-607, 1988

20. Topakian R, Strasak AM, Sonnberger M, Haring HP, Nussbaumer K, Trenkler J and Aichner FT: Timing of stenting of symptomatic carotid stenosis is predictive of 30-day outcome. Eur J Neurol 14: 672-678, 2007.

21. Koton S, Schneider AL, Rosamond WD, Shahar E, Sang Y, Gottesman RF and Coresh J: Stroke incidence and mortality trends in US communities, 1987 to 2011. JAMA 312: 259-268, 2014.

22. Neurology branch of Chinese Medical Association: China cerebrovascular disease prevention and control guide. People's Health Press, Beijing, p5, 2007.
23. van der Vaart MG, Meerwaldt R, Reijnen MM, Tio RA and Zeebregts CJ: Endarterectomy or carotid artery stenting: the quest continues. Am J Surg 195: 259-269, 2008.

24. Ederle J, Featherstone RL and Brown MM: Randomized controlled trials comparing endarterectomy and endovascular treatment for carotid artery stenosis: A Cochrane systematic review. Stroke 40: 1373-1380, 2009.

25. European Stroke Organisation (ESO) Executive Committee and ESO Writing Committee: Guidelines for management of ischaemic stroke and transient ischaemic attack 2008. Cerebrovasc Dis 25: 457-507, 2008.

26. Goldstein LB, Adams R, Alberts MJ, Appel LJ, Brass LM, Bushnell CD, Culebras A, Degraba TJ, Gorelick PB, Guyton JR, et al; American Heart Association/American Stroke Association Stroke Council; Atherosclerotic Peripheral Vascular Disease Interdisciplinary Working Group; Cardiovascular Nursing Council; Clinical Cardiology Council; Nutrition, Physical Activity, and Metabolism Council; Quality of Care and Outcomes Research Interdisciplinary Working Group; American Academy of Neurology: Primary prevention of ischemic stroke: a guideline from the American Heart Association/American Stroke Association Stroke Council: cosponsored by the Atherosclerotic Peripheral Vascular Disease Interdisciplinary Working Group; Cardiovascular Nursing Council; Clinical Cardiology Council; Nutrition, Physical Activity, and Metabolism Council; and the Quality of Care and Outcomes Research Interdisciplinary Working Group: the American Academy of Neurology affirms the value of this guideline. Stroke 37: 1583-1633, 2006.

27. Touzé E, Trinquart L, Chatellier G and Mas JL: Systematic review of the perioperative risks of stroke or death after carotid angioplasty and stenting. Stroke 40: e683-e693, 2009.

28. Kastrup A, Gröschel K, Schnaudigel S, Nägele T, Schmidt F and Ernemann U: Target lesion ulceration and arch calcification are associated with increased incidence of carotid stenting-associated ischemic lesions in octogenarians. J Vasc Surg 47: 88-95, 2008.

29. Bazan HA, Pradhan S, Mojibian H, Kyriakides T and Dardik A: Increased aortic arch calcification in patients older than 75 years: Implications for carotid artery stenting in elderly patients. J Vasc Surg 46: 841-845, 2007.

30. Montorsi P, Caputi L, Galli S, Ciceri E, Ballerini G, Agrifoglio M, Ravagnani P, Trabattoni D, Pontone G, Fabbiocchi F, et al: Microembolization during carotid artery stenting in patients with high-risk, lipid-rich plaque. A randomized trial of proximal versus distal cerebral protection. J Am Coll Cardiol 58 1656-1663, 2011.

31. Mlekusch W, Schillinger M, Sabeti S, Nachtmann T, Lang W, Ahmadi R and Minar E: Hypotension and bradycardia after elective carotid stenting: Frequency and risk factors. J Endovasc Ther 10: 851-859, discussion 860-861, 2003.

32. Taha MM, Toma N, Sakaida H, Hori K, Maeda M, Asakura F, Fujimoto M, Matsushima S and Taki W: Periprocedural hemodynamic instability with carotid angioplasty and stenting. Surg Neurol 70: 279-285, discussion 285-286, 2008.

33. Jeon JS, Sheen SH and Hwang G: Hemodynamic instability during carotid angioplasty and stenting-relationship of calcified plaque and its characteristics. Yonsei Med J 54: 295-300, 2013.

34. Lian X, Lin M, Zhu S, Liu W, Li M, Sun W, Yin Q, Xu G, Zhang R and Liu X: Risk factors associated with haemodynamic depression during and after carotid artery stenting. J Clin Neurosci 18: 1325-1328, 2011. 